\title{
$\beta$-Amyloid 25-35 Peptide Reduces the Expression of Glutamine Transporter SAT1 in Cultured Cortical Neurons
}

\author{
Doungjai Buntup • Øivind Skare · Tom Tallak Solbu · Farrukh A. Chaudhry • \\ Jon Storm-Mathisen · Wipawan Thangnipon
}

Accepted: 5 October 2007 / Published online: 1 November 2007

(C) Springer Science+Business Media, LLC 2007

\begin{abstract}
Amyloid $(\mathrm{A} \beta)$ peptides may cause malfunction and death of neurons in Alzheimer's disease. We investigated the effect of $\mathrm{A} \beta$ on key transporters of amino acid neurotransmission in cells cultured from rat cerebral cortex. The cultures were treated with $\mathrm{A} \beta(25-35)$ at 3 and $10 \mu \mathrm{M}$ for 12 and $24 \mathrm{~h}$ followed by quantitative analysis of immunofluorescence intensity. In mixed neuronal-glial cell cultures (from P1 rats), $\mathrm{A} \beta$ reduced the concentration of system A glutamine transporter 1 (SAT1), by up to $50 \%$ expressed relative to the neuronal marker microtubuleassociated protein 2 (MAP2) in the same cell. No significant effects were detected on vesicular glutamate transporters VGLUT1 or VGLUT2 in neurons, or on glial
\end{abstract}

Special issue article in honor of Dr. Frode Fonnum.

D. Buntup · W. Thangnipon $(\bowtie)$

Neuro-Behavioural Biology Center, Institute of Science and

Technology for Research and Development, Mahidol University,

Salaya, Nakorn-pathom 73170, Thailand

e-mail: grwtn@mahidol.ac.th

$\varnothing$. Skare

Department of Biostatistics, Institute of Basic Medical Sciences, University of Oslo, P.O. Box 1105, Blindern, Oslo N-0317,

Norway

T. T. Solbu · F. A. Chaudhry · J. Storm-Mathisen

Department of Anatomy, Institute of Basic Medical Sciences, University of Oslo, P.O. Box 1105, Blindern, Oslo N-0317, Norway

T. T. Solbu · F. A. Chaudhry · J. Storm-Mathisen Centre for Molecular Biology and Neuroscience, University of Oslo, P.O. Box 1105, Blindern, Oslo N-0317, Norway

T. T. Solbu · F. A. Chaudhry

The Biotechnology Centre of Oslo, University of Oslo, P.O. Box

1125, Blindern, Oslo N-0317, Norway system $\mathrm{N}$ glutamine transporter 1 (SN1). In neuronal cell cultures (from E18 rats), A $\beta(25-35)$ did not reduce SAT1 immunoreactivity, suggesting that the observed effect depends on the presence of astroglia. The results indicate that $\mathrm{A} \beta$ may impair neuronal function and transmitter synthesis, and perhaps reduce excitotoxicity, through a reduction in neuronal glutamine uptake.

Keywords $\beta$-Amyloid peptide (25-35) - Cell culture · Alzheimer's disease · Vesicular glutamate transporter . Glutamine transporter

\section{Introduction}

Alzheimer's disease (AD) is a neurodegenerative disorder, leading to progressive loss of memory, impaired cognition and debilitation. It affects about $2 \%$ of the population in industrialized countries, the risk of having the disease increasing rapidly with age beyond 70 , hence the prevalence of $\mathrm{AD}$ will increase several fold in the coming decades $[1,2] . \mathrm{AD}$ is characterized by extracellular deposits of $\beta$-amyloid $(\mathrm{A} \beta)$, associated with dystrophic neuronal processes, degenerating and apoptotic neurons showing neurofibrillary tangles (i.e. aggregates of hyperphosphorylated tau protein filaments).

The neurotoxic effects of $\mathrm{A} \beta$ and some of its fragments have been demonstrated in in vitro primary cultures from human and rat cortical neurons, in hippocampal slice cultures and following in vivo injection into various areas of the rat and monkey brain [3]. $\mathrm{A} \beta$ peptides cause neuronal cell death and also increase the vulnerability of neurons to excitotoxicity [4]. $\mathrm{A} \beta(25-35)$ has been frequently used in investigations of $\mathrm{A} \beta$ properties as a less expensive and more easily handled substitute for the native full-length peptide, 
$\mathrm{A} \beta(1-42)$, the latter being implicated in the pathogenesis of AD. Implicit in these experiments is the assumption that the mechanism of action of $\mathrm{A} \beta(25-35)$ is similar to that of $\mathrm{A} \beta(1-42)$. Indeed, $\mathrm{A} \beta(25-35)$ mimics the toxicological and aggregational properties of the full-length peptide, though these characteristics are increased; i.e., the shorter peptide is more toxic to cultured neurons, exhibits toxicity earlier, causes more membrane protein oxidation, and aggregates faster than the native $\mathrm{A} \beta(1-42)$ [5].

The amino acids glutamine and glutamate are essential for brain metabolism and function. Glutamate is the major excitatory transmitter of the brain [6], particularly in the cerebral cortex and hippocampus, and therefore has important roles in cognition, learning and memory. After synaptic release, it is taken up mainly into astroglia by glutamate transporters [7]. Perturbed glutamate uptake may cause excitotoxicity and is implicated in the pathogenesis of $\mathrm{AD}$ [7]. Once accumulated into astrocytes, glutamate is converted to the non-excitatory amino acid glutamine by the glial enzyme glutamine synthetase. Glutamine is then returned to neurons via glutamine transporters of system $\mathrm{N}$ (SNs) and system A (SATs) and used as a source for the synthesis of glutamate through its conversion by phosphate-activated glutaminase [8, 9]. Glutamate is packaged for exocytosis by vesicular glutamate transporters (VGLUTs) [10, 11], or used as precursor of GABA in GABAergic neurons.

The aim of this study was to explore the effects of $\mathrm{A} \beta(25-35)$ treatment on main transporters implicated in sustaining synaptic release of amino acids, i.e. the glutamine transporters $\mathrm{SN} 1$ and SAT1 and the vesicular glutamate transporters VGLUT1 and VGLUT2, in two different in vitro models: neuronal cell cultures and mixed cell cultures of rat cerebral cortex.

\section{Experimental procedures}

\section{Cell cultures}

Neuronal cultures were prepared from embryos of Wistar rats at day 18 of gestation (E18) using a modification of an earlier described method [12]. In brief, the neocortices of embryos were dissociated by treatment with $0.25 \%$ trypsin/calcium-magnesium-free buffer/EDTA for $10 \mathrm{~min}$ at $37^{\circ} \mathrm{C}$. The suspension was settled for $2 \mathrm{~min}$ at $2,000 \times g$ and then the cells were mixed in DMEM, triturated and passed through a cell strainer. The cells were cultured in DMEM supplemented with 2\% B27 and 1\% GlutaMAX I. The cells were plated on poly-L-lysine $(50 \mu \mathrm{g} / \mathrm{ml})$ coated glass coverslips at a density $0.5 \times 10^{6}$ cells $/ \mathrm{ml}$ and the cultures were maintained in a humidified atmosphere of $5 \%$ $\mathrm{CO}_{2} / 95 \%$ air at $37^{\circ} \mathrm{C}$ for 5 days in vitro before incubation with peptide.
Primary mixed cell cultures were prepared from the cerebral cortices of 1-day-old (P1) neonatal Wistar rats and plated on poly-L-lysine-treated coverslips (see above). The dissociated cortical cells were suspended in Neurobasal-A medium containing 2\% B27 and 1\% GlutaMAX I and maintained as described for E18 cells.

Rats were kept according to nationally and internationally approved conditions. Culture media and reagents were from Gibco, Invitrogen Corporation (Carlsbad, CA, USA).

Incubation with $\mathrm{A} \beta$ peptide

Neuronal and mixed cell cultures were treated with $\mathrm{A} \beta(25-$ 35) (Sigma-Aldrich, St.Louis, MO, USA) at final concentrations of 3 and $10 \mu \mathrm{M}$, for 12 or $24 \mathrm{~h}$. In this study the B27-containing medium was removed from the cultures at day 6. Cells were washed twice with DMEM or Neurobasal-A medium and then incubated in medium for 12 and $24 \mathrm{~h}$ in the absence or presence of $\mathrm{A} \beta(25-35)$. Controls received no peptide.

\section{Antibodies}

Antibody to SAT1 was raised in rabbit to a fusion protein corresponding to the N-terminal amino acids 1-71 of SAT1 (aka GlnT, [13]; aka SA2, [14]), and affinity purified and characterized (T.T. Solbu \& F.A. Chaudhry, to be published) as described for SN1 [15]. A GST-fusion peptide was made by cloning the first 213 base pairs from SAT1 into a pGex3X vector (Amersham Biosciences, GE Healthcare, Oslo, Norway). Using primers $5^{\prime}$ - cccgggatccttatgatgcatttcaaa- $3^{\prime}$ and $5^{\prime}$-gcggaattcggttgttcctggaat- $3^{\prime}$ (Eurogentech, Herstal, Belgium), the $5^{\prime}$ end of SAT1 was amplified by PCR and ligated into pGex3X using BamH1 and EcoR1 (New England Biolabs, Ipswich, MA, USA). GST-SAT1 was purified on Gluthatione Sepharose 4B as prescribed by the manufacturer (Amersham Biosciences; "GST Gene Fusion System Handbook"). Antibodies to other transporters were prepared and characterized as described (VGLUT1 and VGLUT2: [16]; SN1: [15]). Antibodies to marker proteins [glial fibrillary acidic protein (GFAP), anti-microtubule associated protein2A\&B (MAP2)], raised in mice, were from Sigma-Aldrich. Secondary antibodies were Alexa Fluor 488 goat anti-mouse IgG and Alexa Fluor 555 goat anti-rabbit IgG from Molecular Probes (Eugene, Oregon, USA).

Immunocytochemical staining

Following exposure to peptide, cells attached to 14-mm glass coverslips were fixed with formaldehyde $(4 \%$ 
paraformaldehyde, freshly depolymerized, in $0.1 \mathrm{M}$ sodium phosphate buffer $\mathrm{pH} \mathrm{7.4)}$ for $20 \mathrm{~min}$ at room temperature followed by three washes in phosphate buffered saline (10 mM phosphate buffer in $150 \mathrm{mM} \mathrm{NaCl}$ ) and then blocked with blocking buffer [10\% (v/v) newborn calf serum (NCS), 3\% (w/v) bovine serum albumin (BSA), $0.5 \%(\mathrm{w} / \mathrm{v})$ Triton X-100 in Tris buffered saline (TBS) $\mathrm{pH}$ 7.4] for $1 \mathrm{~h}$ at room temperature. After blocking, sections were incubated with the primary antibodies (antiVGLUT1 1:3,000; anti-VGLUT2 1:3,000; anti-SAT1 $2 \mathrm{mg} / \mathrm{ml}$; anti-SN1 $0.3 \mathrm{mg} / \mathrm{ml}$; anti-MAP2 1:200; GFAP 1:500; dilutions optimized in pilot experiments) in primary antibody solution [3\% (v/v) NCS, $1 \%$ BSA, $0.5 \%$ Triton $\mathrm{X}-100,0.05 \% \mathrm{NaN}_{3}$ in TBS pH 7.4] overnight at $4^{\circ} \mathrm{C}$, and then the cells were washed and incubated with the secondary antibodies conjugated to Alexa Fluor 488 and Alexa Fluor 555 for $1 \mathrm{~h}$ at room temperature. The coverslips were then washed three times in fresh antibody solution. Cells on coverslips were sealed to microscopic slides with Fluoromout G water based (Southern Biotechnology Associates, Birmingham, AL, USA).

\section{Quantitative analysis of immunoreactivities}

Dual immunofluorescence for VGLUT1, VGLUT2, SAT1, SN1, GFAP and MAP2 was examined and quantified with a Zeiss Axioplan 2 microscope equipped with an LSM 5 PASCAL confocal unit, LSM software and a 40x/1.3 oil PlanApo objective (Carl Zeiss, Heidelberg, Germany). We used standard FICT-rhodamine filter settings. Other settings were optimized initially, and held constant throughout the study so that all sections were digitized under the same conditions of illumination. Care was taken to use similar times for orientation (with conventional fluorescence) and adjustments in all preparations, in order to avoid differences in photobleaching. The cells were selected on the criterion that they showed representative morphology (Fig. 1) and strong immunoreactivity for MAP2 (neurons) or for SN1 (in the case of astroglia). The cell body and the most proximal parts of the processes were outlined for digitalization of area and of fluorescence intensity for two immunoreactivities (MAP2 and a transporter). The analysis was performed without notice of the treatment group; the lack of difference in neuronal area between treatment groups suggests that sampling bias did not interfere with the results obtained. Fluorescence intensity is expressed in arbitrary units, without subtraction of background. For each glass coverslip, five fields were randomly sampled and two cells quantified per field, i.e. 10 cells, to obtain an average value for each immunoreactivity and experiment.
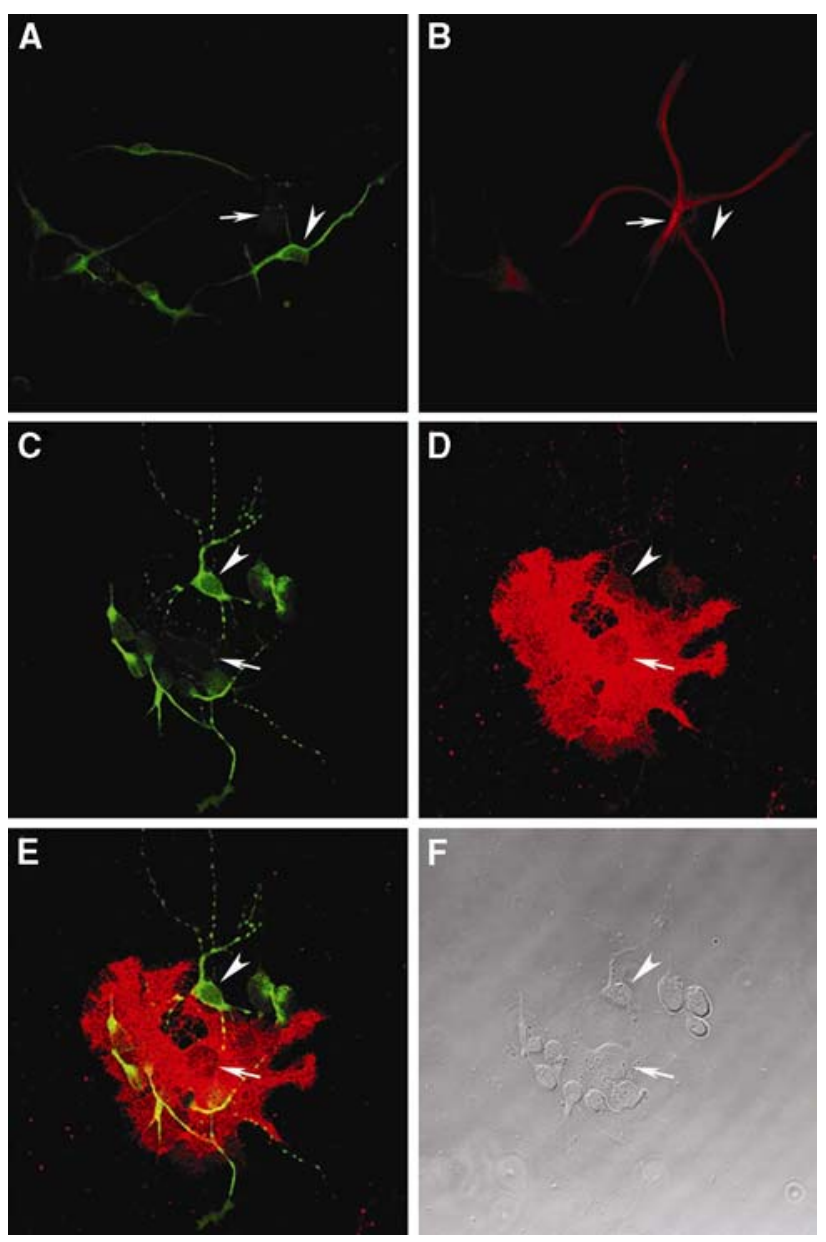

Fig. 1 Classification of cell types. (a, c, e) MAP2 positive (green) neuronal cells (arrowheads). (b) GFAP positive (red) astroglial cell (arrow, same field as a). (d, e) SN1 positive (red) astroglial cell (arrow, same field as c); SN1 (in contrast to GFAP) reveals the entire extent of the astrocyte. (e) MAP2 and SN1 immunoreacivities superimposed. (f) DIC image (same field as c-e). Arrowheads and arrows indicate the positions of the nuclei of sample neurons and astrocytes, respectively. Note that all of the 10 cells situated on or next to the astrocyte in (f) are identified as MAP2 positive neurons in (c). Double immunofluorescence observed by confocal microscopy in mixed cell cultures from rat cortex at P1

\section{Data analysis}

Data are expressed as mean \pm standard error of the mean (SEM) of four different experiments (four different sets of cultures prepared at four different times) for P1 and E18 cultures. Advanced statistical analysis was performed according to a linear mixed model regression analysis, using the function lme of the nlme package [17] in the statistical software R (http://www.r-project.org/). The fundamental assumptions of the model are that the observations are normally distributed with a variance varying only with protein type, and that they are uncorrelated when they come from different experiments. Log (for P1 data) or square root (for E18 data) transformations were performed 
as quantile-quantile plots showed these transformations to give the best fits to the Gaussian distribution.

\section{Results}

Cell types

Cell types were characterized in control cultures incubated without $\mathrm{A} \beta$. In mixed neuronal-glial cultures (from cortex of P1 rat pups), neurons and astroglia were clearly identified by immunoreactivity for the markers MAP2 and GFAP, respectively (Fig. 1a, b). Double labelling for MAP2 and system $\mathrm{N}$ glutamine transporter $\mathrm{SN} 1$, localized in the membranes of astroglia [15], again showed the two immunoreactivities in separate cells (Fig. 1c-e), SN1 displaying the full extension of protoplasmatic-like astrocytes (i.e. beyond the parts pervaded by GFAP filaments, Fig. 1a, b). The MAP2 expressing cells show the morphological characteristics of neurons and usually grow in close apposition to astroglia (Fig. 1a-f). In neuronal cultures (from E18 rats), MAP2 positive neurons had similar morphology, but no GFAP positive astroglial cells were found (not shown).

Double labelling for MAP2 and different transporter proteins showed the vesicular glutamate transporters VGLUT1 or VGLUT2 to be in neurons. A slight signal for SN1 in neurons was close to background levels. The system A glutamine transporter SAT1 was mainly localized in neurons but slight immunoreactivity was seen in some astroglial cells (Fig. 2a, b). In the neurons, SAT1 staining was strong in central areas of the perikaryon and pervaded the processes.

Survey of preparations double stained for MAP2 and either VGLUT1 or VGLUT2 indicated that a large proportion of the neurons were VGLUT immunoreactive. Similarly, double staining for MAP2 and SAT1 suggested that most of the neurons in the cultures contained SAT1.

\section{Effects of $\mathrm{A} \beta$ in mixed cell cultures}

Cortical mixed cell cultures (P1) were exposed to A $\beta$. After day 5 in vitro cell cultures were incubated with 3 or $10 \mu \mathrm{M}$ of $\mathrm{A} \beta(25-35)$ for 12 or $24 \mathrm{~h}$ and then processed for immunohistochemistry and quantitative analyses. Neurons with typical morphology and strong MAP2 staining were seen also after exposure to $\mathrm{A} \beta$ (Fig. $2 \mathrm{c}$, d). The cells appeared morphologically similar to those in control cultures (Figs. 1, 2a, b). Transporter immunoreactivities were quantitatively analysed (Fig. 3a, b) in neurons with typical morphology and clear MAP2 immunoreactivity. In addition, SN1 was quantified in cells with typical astroglial morphology according to SN1 immunofluorescence. All immunoreactivities showed a tendency for decline on exposure to $\mathrm{A} \beta(25-35)$, at $12 \mathrm{~h}$ as well as at $24 \mathrm{~h}$, but this attained statistical significance only for SAT1. (The low SN1 level in neurons is close to background and may not be biologically significant.) The size of the central areas of the cells, over which the fluorescence intensities were recorded, was independent of peptide exposure in the neurons and only slightly reduced (by about $16 \%$ ) in the glia (Fig. 3c, d). This indicates that, in the neurons recorded, $\mathrm{A} \beta$ treatment did not cause general impairment resulting in a reduction of cell size.

To adjust for differences in the condition of the individual cell, transporter immunoreactivity was expressed relative to the simultaneously recorded MAP2 immunoreactivity of the same cell (Fig. 3e, f). In this analysis, the effect of $\mathrm{A} \beta$ on SAT1 stood out even more clearly, showing the largest percentage changes, whereas there were no statistically significant effects of $\mathrm{A} \beta$ on VGLUT1, VGLUT2 or SN1 in neurons, neither on SN1 in astroglia. After both 12 and $24 \mathrm{~h}$ incubation, SAT1 was reduced to about two thirds of the control level by $3 \mu \mathrm{M}$ of $\mathrm{A} \beta$, and about one half by $10 \mu \mathrm{M}$ of $\mathrm{A} \beta$. As statistical tests showed similar effects at 12 and $24 \mathrm{~h}$, the data were reanalysed statistically with a simplified linear mixed model assuming the same fixed effect at 12 and $24 \mathrm{~h}$. This analysis gave higher statistical significance for the effect on SAT1 $(P=0.0009$ at $3 \mu \mathrm{M}, P=0.0000$ at $10 \mu \mathrm{M})$ than did the full model reported in Fig. 3. It confirmed the lack of effect on the other transporters $(P>0.2)$, and the lack of effect on perikaryal size $(P>0.1)$, except for the size of SN1 positive glia $(P<0.03$ for $3 \mu \mathrm{M}, P<0.01$ for $10 \mu \mathrm{M})$.

Lack of effect of $\mathrm{A} \beta$ in neuronal cell cultures

Cultured cortical neurons (E18) were exposed to $\mathrm{A} \beta(25-$ $35)$ in the same way as for P1 mixed cell cultures. MAP2 positive neurons had a similar appearance as in $\mathrm{P} 1$ cultures, in controls as well as after exposure to $\mathrm{A} \beta$ (Fig. 4a-d). Quantification of the fluorescence intensities (Fig. 5) indicated slight effects of $\mathrm{A} \beta$ on SAT1 and SN1 (Fig. 5a, b), but not on the other transporters, or on cell area (Fig. 5c, d). However, when the fluorescence intensity for transporter was normalized to that for MAP2 in the same cell, there were no significant effects of $\mathrm{A} \beta$, except on SN1 (which in neurons is very low and of questionable biological significance). Statistical tests suggested no difference between 12 and $24 \mathrm{~h}$ or between 3 and $10 \mu \mathrm{M}$. When statistically analysed according to a simplified linear mixed model, assuming the same fixed effect for the different incubation times and concentrations of $\mathrm{A} \beta$, there were no significant effects of $\mathrm{A} \beta$ on any of the transporter immunoreactivities $(P \geq 0.07)$.

Finally, to test more directly whether the results differed between the two types of culture used, the MAP2 

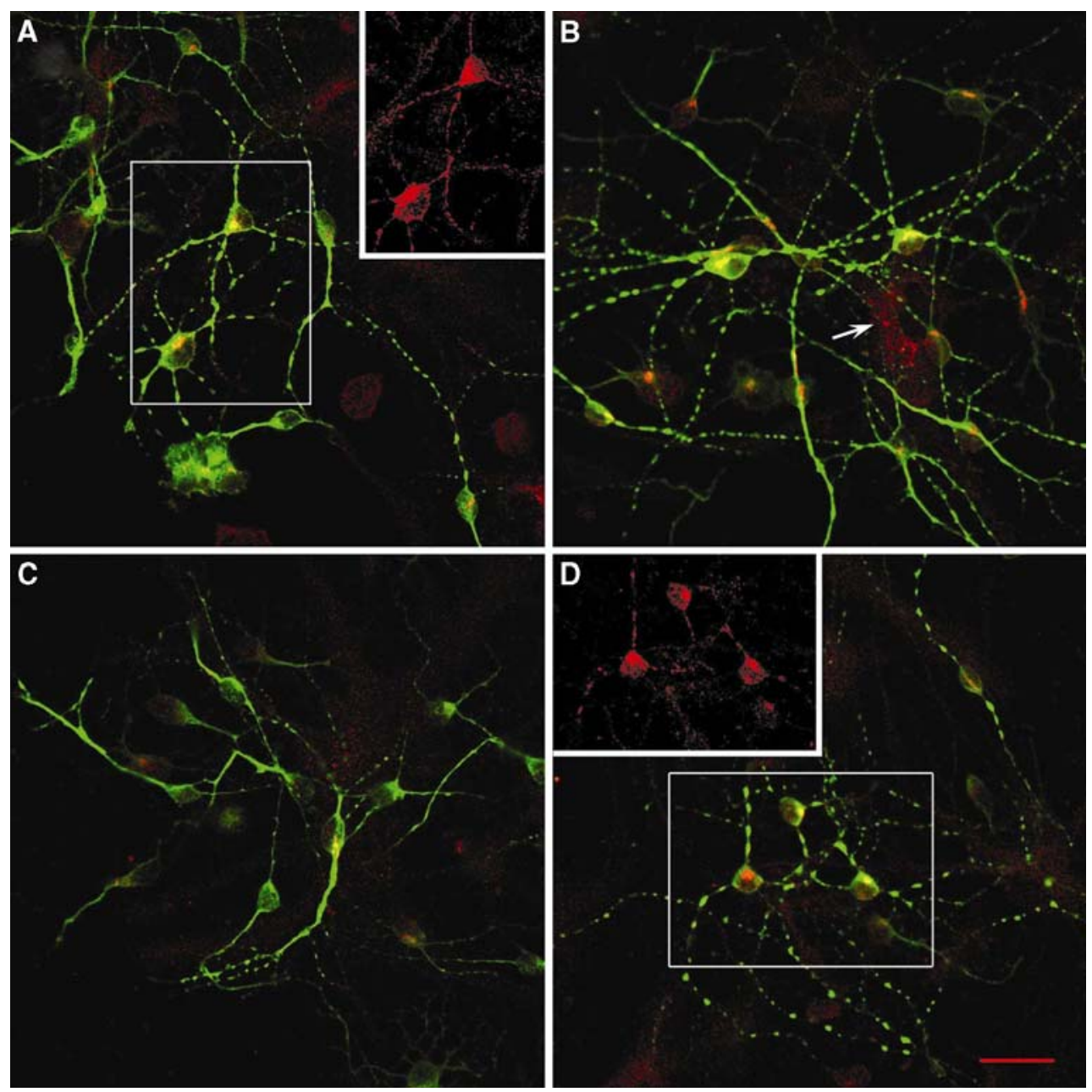

Fig. 2 SAT1 (red) colocalized (yellow-orange) with MAP2 (green) in neurons in P1 cortical mixed cell cultures. Images show sample cells, insets display framed areas separately for SAT1 immunoreactivity. Cultures were exposed to control medium for $12 \mathrm{~h} \mathrm{(a)} \mathrm{or} 24 \mathrm{~h}$ (b), or to $10 \mu \mathrm{M}$ of $\mathrm{A} \beta(25-35)$ for $12 \mathrm{~h}(\mathbf{c})$ or $24 \mathrm{~h}(\mathbf{d})$. SAT1 is expressed in the soma and processes of neurons, as revealed by double labelling with antibodies to the somatodendritic neuronal

normalized data for P1 and E18 cultures were combined into one linear mixed model for regression analysis. The fixed effect was now defined as the expected difference in effect of $\mathrm{A} \beta$ exposure between $\mathrm{P} 1$ and $\mathrm{E} 18$ cultures. Assuming the same fixed effect at 12 and $24 \mathrm{~h}$, the effect on SAT1 was significantly different between neurons in P1 and E18 cultures $(P=0.016$ for $3 \mu \mathrm{M}, P=0.010$ for $10 \mu \mathrm{M}$ of $\mathrm{A} \beta$ ) but those on the other transporters were not $(P>0.3)$. Assuming similar effect at the two concentrations of $\mathrm{A} \beta$, the significance level was even higher (SAT1 $P=0.004$; other transporters $P>0.2$ ).

\section{Discussion}

The main finding of the present study is that $\mathrm{A} \beta$ causes a reduction in neuronal SAT1, a transporter thought to be

marker MAP2 shown superimposed on SAT1. Foci of strong perikaryal immunoreactivity suggest intracellular stores of SAT1. (Slight SAT1 immunoreactivity occurs also in glia-like perikarya (b, centre right, arrow), immunonegative for MAP2.) The immunoreactive cells were morphologically similar in the different conditions (ad). Scale bar $=20 \mu \mathrm{m}$

essential for neuronal uptake of glutamine $[9,13,14]$ to support neuronal function including the formation of neurotransmitter in glutamatergic as well as GABAergic neurons. The transmitter of the neurons loosing SAT1 was not directly identified, but as double labelling for MAP2 with VGLUTs and MAP2 with SAT1 both showed major proportions of double labelled cells, they are likely to comprise glutamatergic neurons.

The concentrations of $\mathrm{A} \beta$ were selected on the basis of literature data to be effective but with low to moderate toxicity levels [12]. To further minimize confounding influence of indiscriminate toxic damage, the immunoreactivities were measured in individual cells that showed near normal morphology and near normal immunoreactivity for the neuronal marker MAP2. Therefore, unlike in biochemical studies of whole cultures, cells dying or dead 
A

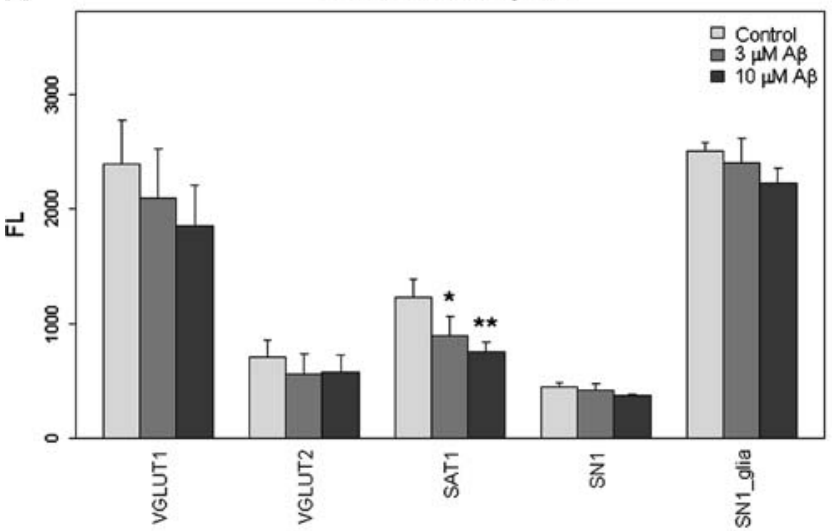

C
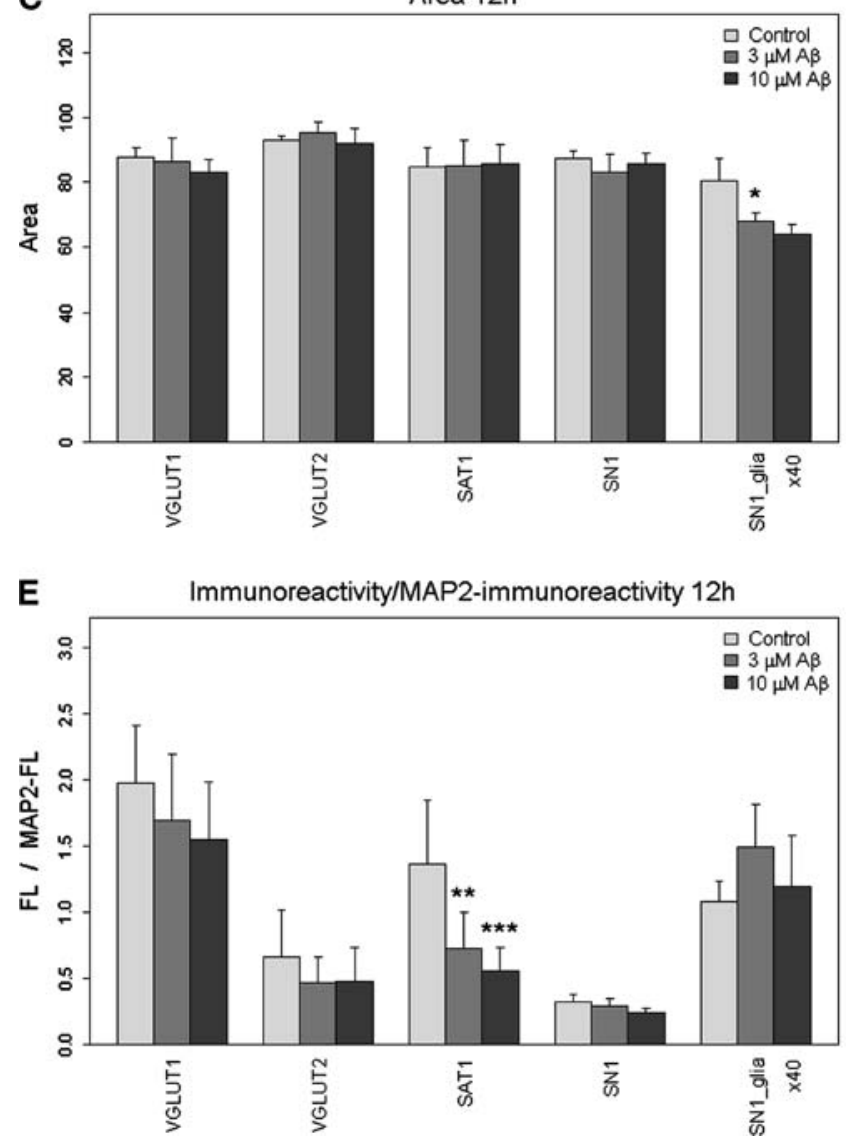

Fig. 3 A $\beta$ induced a reduction of SAT1 immunoreactivity in neurons in P1 mixed cortical cell cultures. Transporter immunoreactivities (arbitrary units of fluorescence intensity, FL) and areas $\left(\mu \mathrm{m}^{2}\right)$ were recorded in individual cells in cultures incubated with 0,3 or $10 \mu \mathrm{M}$ $\mathrm{A} \beta(25-35)$ for $12 \mathrm{~h}(\mathbf{a}, \mathbf{c}, \mathbf{e})$ or $24 \mathrm{~h}(\mathbf{b}, \mathbf{d}, \mathbf{f})$. Areas $(\mathbf{c}, \mathbf{d})$ comprising the perikaryon and proximal neurites were outlinded for recording of an average fluorescence intensity in each cell. To cancel out random differences between cells, transporter immunoreactivity was also expressed relative to MAP2 immunoreactivity in the same cell $(\mathbf{e}, \mathbf{f})$. All values are for neurons, except for SN1, which was recorded also
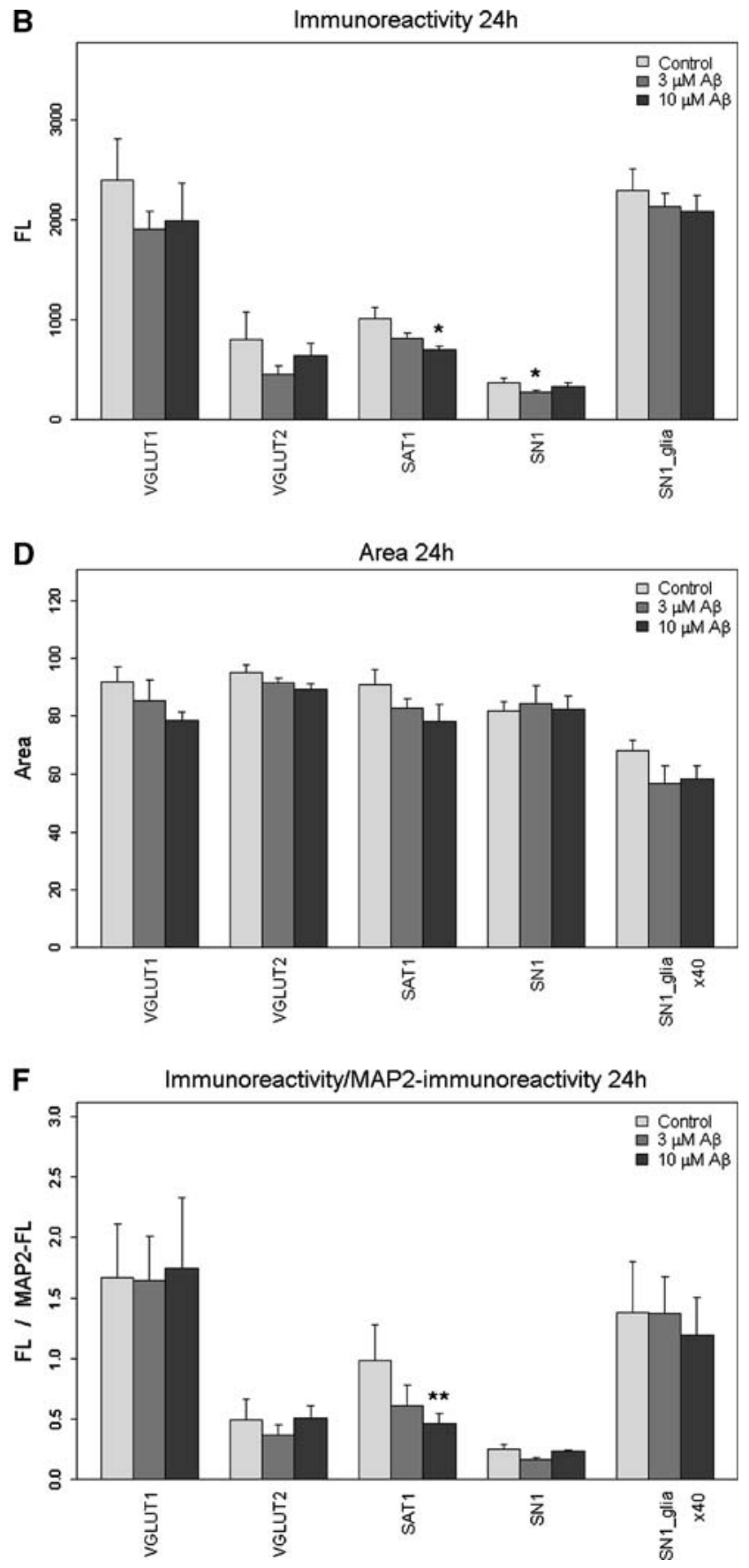

in astroglial cells (area and relative immunoreactivity for SN1 in glia were 40 times the displayed numbers). Background was not subtracted; the low values for SN1 in neurons are close to nonimmunoreactive background. Columns show mean + SEM of measurements from four independent experiments $(n=4)$, in each experiment 10 cells were averaged. Asterisks indicate a statistically significant difference compared with control $(* P \leq 0.05$, **P $\leq 0.01, * * * P \leq 0.001)$ in a linear mixed model regression analysis (statistical software R). Only SAT1 showed a robust effect of A $\beta$ 

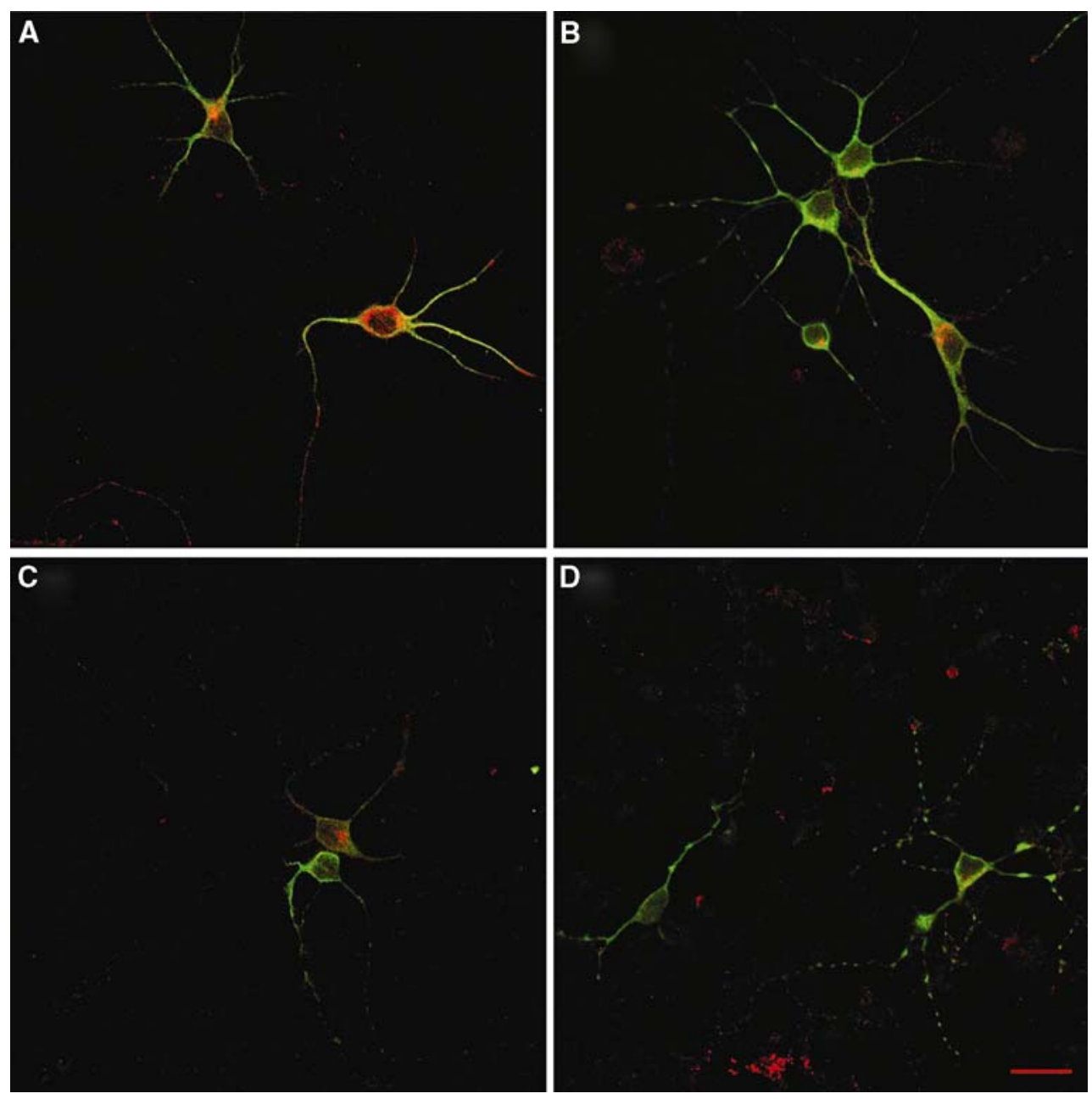

Fig. 4 SAT1 (red) colocalized (yellow-orange) with MAP2 (green) in neurons in E18 cortical neuronal cell cultures. Sample neurons are shown. The immunoreactive cells were morphologically similar to those in P1 cultures (Fig. 2). No glia-like cells were present. Cells

due to toxic effects of $\mathrm{A} \beta$ [5] were excluded from analysis. Further, random variation in the condition of cells was corrected for by expressing the transporter immunoreactivities relative to that of MAP2, measured in the same cell by double immunofluorescence. $\mathrm{A} \beta$ peptides have been found to induce proteolysis of MAP2 in cultured mouse cortical neurons [18], but in the cells sampled in the present study such proteolysis must have been minor, as the relation between the values for the different transporters and conditions were similar whether expressed as fluorescence units or relative to MAP2 fluorescence units.

The effect of $\mathrm{A} \beta$ on neuronal SAT1 was observed in cultures containing neurons in close apposition to astroglial cells, but not in neuronal cultures without glia. This suggests that $\mathrm{A} \beta$ does not produce the effect by acting directly on the neurons, but via the glial cells, an idea gaining some support from the observed reduction in astroglial cell size. However, as the astrocytes had only slightly reduced size were incubated in the absence $(\mathbf{a}, \mathbf{b})$ or presence of $10 \mu \mathrm{M} \mathrm{A} \beta(25-35)$ (c, d) for $12 \mathrm{~h}(\mathbf{a}, \mathbf{c})$ or $24 \mathrm{~h}$ (b, d). Labelling was strong in the perinuclear region and processes of neurons. Scale bar $=20 \mu \mathrm{m}$

and normal levels of their marker protein SN1, the changes in neuronal SAT1 cannot be ascribed simply to toxic damage in the glia.

The findings are consistent with previous reports that the presence of astrocytes enhances $\mathrm{A} \beta$ induced neurotoxicity in hippocampal cell cultures [19] and that the presence of reactive astrocytes is a characteristic feature of the lesions in AD brain [20]. Notwithstanding, the effect on neuronal SAT1 cannot be ascribed simply to an indiscriminate toxic influence on the neurons from the $\mathrm{A} \beta$ exposed glia, because there was no similar effect on VGLUTs, and because the effect was observed in neurons with apparently normal morphology and normal expression of the neuronal marker MAP2. Could the reduced level of neuronal SAT1 contribute to the impaired resistance of neurons to $\mathrm{A} \beta$ when cultured in the presence of glia? While this does not appear likely, the observed 50\% reduction in glutamine transporter level might possibly be critical in certain conditions. 

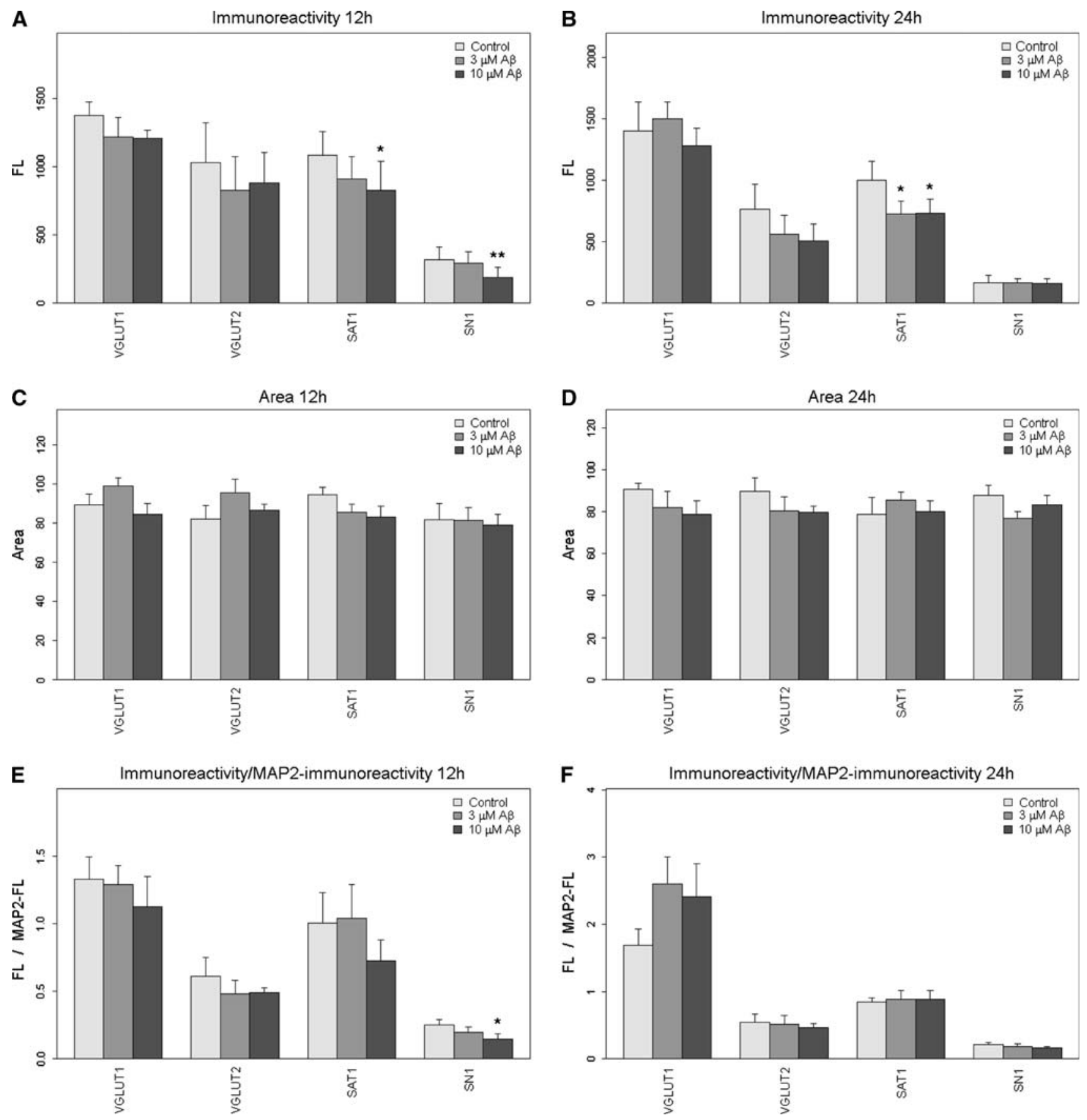

Fig. 5 Lack of effect of A $\beta$ on SAT1 immunoreactivity in E18 cortical neuronal cells (cultured without glia). Data were obtained and presented as in Fig. 3

The mechanism by which astroglial cells cause changes in neuronal expression of SAT1 must be determined by future research. As free radicals and oxidative stress play important parts in $\mathrm{AD}$ [21], it is interesting that crotonaldehyde accumulates in reactive astrocytes in $\mathrm{AD}$ brain [22]. Astrocytes mediate $\mathrm{A} \beta$ induced neuronal death through oxidative stress [23]. One possible mediator of the observed glia-neuron influence is BDNF signalling, which is impaired in cortical neuron cultures exposed to $\mathrm{A} \beta[24$, 25], although $\mathrm{A} \beta$ may affect this signalling cascade without the participation of glia [24]. An attractive possibility is offered by the observation that $\mathrm{A} \beta(25-35)$ causes astrocytes to produce nerve growth factor (NGF), thereby inducing tau hyperphosphorylation and reduced survival in hippocampal neurons [26]. As the effect is exacerbated by NO, this mechanism may offer a link with the effects of free 
radicals in $\mathrm{AD}$ pathology. It fits with the recent observation that reduction of endogenous tau protects against $\mathrm{A} \beta$ toxicity in a mouse model of AD [27].

The mechanism of vulnerability of neurons in $\mathrm{AD}$ appears to involve excitotoxicity [4]. The increase in glial glutamate transporters induced by $\mathrm{A} \beta[28]$ may represent a measure to limit excitotoxic damage. Similarly, the reduced neuronal levels of SAT1 observed here after exposure to $\mathrm{A} \beta$ may limit the production of glutamate, but also of GABA, for synaptic release. It might represent an adaptation to curb excitotoxicity in $\mathrm{AD}$, but might concomitantly impair synaptic function.

Acknowledgements This work was supported by the Thailand Research Fund through the Royal Golden Jubilee Ph.D. Program (Grant No. PHD/0230/2545), and by the Research Council of Norway.

\section{References}

1. Mattson MP (2004) Pathways towards and away from Alzheimer's disease. Nature 430:631-639

2. Goedert M, Spillantini MG (2006) A century of Alzheimer's disease. Science 314:777-781

3. Kasa P, Papp H, Kasa Jr P et al (2004) Effects of amyloid-beta on cholinergic and acetylcholinesterase-positive cells in cultured basal forebrain neurons of embryonic rat brain. Brain Res 998:73-82

4. Mattson MP, Cheng B, Davis D et al (1992) $\beta$-Amyloid peptides destabilize calcium homeostasis and render human cortical neurons vulnerable to excitotoxicity. J Neurosci 12:376-389

5. Varadarajan S, Kanski J, Aksenova M et al (2001). Different mechanisms of oxidative stress and neurotoxicity for Alzheimer's $\mathrm{A} \beta(1-42)$ and $\mathrm{A} \beta(25-35)$. J Am Chem Soc 123:5625-5631

6. Fonnum $\mathrm{F}$ (1984) Glutamate: a neurotransmitter in mammalian brain. J Neurochem 42:1-11

7. Danbolt NC (2001) Glutamate uptake. Prog Neurobiol 65:1-105

8. Armano S, Coco S, Bacci A et al (2002) Localization and functional relevance of system A neutral amino acid transporters in cultured hippocampal neurons. J Biol Chem 277:10467-10473

9. Chaudhry FA, Reimer RJ, Edwards RH (2002) The glutamine commute: take the $\mathrm{N}$ line and transfer to the A. J Cell Biol 157:349-355

10. Fremeau RT Jr, Voglmaier S, Seal RP et al (2004) VGLUTs define subsets of excitatory neurons and suggest novel roles for glutamate. Trends Neurosci 27:98-103

11. Shigeri Y, Seal RP, Shimamoto K (2004) Molecular pharmacology of glutamate transporters, EAATs and VGLUTs. Brain Res Rev 45:250-265

12. Tong L, Thornton PL, Balazs $\mathrm{R}$ et al (2001) $\beta$-amyloid-(1-42) impairs activity-dependent cAMP-response element-binding protein signaling in neurons at concentrations in which cell survival is not compromised. J Biol Chem 276: 17301-17306

13. Varoqui H, Zhu H, Yao D et al (2000) Cloning and functional identification of a neuronal glutamine transporter. J Biol Chem 275:4049-4054

14. Chaudhry FA, Schmitz D, Reimer RJ et al (2002). Glutamine uptake by neurons: interaction of protons with system A transporters. J Neurosci 22:62-72

15. Boulland JL, Osen KK, Levy LM et al (2002) Cell-specific expression of the glutamine transporter SN1 suggests differences in dependence on the glutamine cycle. Eur J Neurosci 15: $1615-1631$

16. Fremeau RT, Troyer MD, Pahner I et al (2001) The expression of vesicular glutamate transporters defines two classes of excitatory synapse. Neuron 31:247-260

17. Pinheiro JC, Bates DM (2002) Mixed effects models in S and SPLUS. Springer, New York

18. Fifre A, Sponne I, Koziel V et al (2006) Microtubule-associated protein MAP1A, MAP1B, and MAP2 proteolysis during soluble amyloid $\beta$-peptide-induced neuronal apoptosis. Synergistic involvement of calpain and caspase-3. J Biol Chem 281:229-240

19. Domenici MR, Paradisi S, Sacchetti B et al (2002) The presence of astrocytes enhances beta amyloid-induced neurotoxicity in hippocampal cell cultures. J Physiol Paris 96:313-316

20. Meda L, Baron P, Scarlato G (2001) Glial activation in Alzheimer's disease: the role of $\mathrm{A} \beta$ and its associated proteins. Neurobiol Aging 22:885-893

21. Pratico D, Delanty N (2000) Oxidative injury in diseases of the central nervous system: focus on Alzheimer's disease. Am J Med 109:577-585

22. Kawaguchi-Niida M, Shibata N, Morikawa S et al (2006) Crotonaldehyde accumulates in glial cells of Alzheimer's disease brain. Acta Neuropathol 111:422-429

23. Abramov AY, Canevari L, Duchen MR (2004) $\beta$-Amyloid peptides induce mitochondrial dysfunction and oxidative stress in astrocytes and death of neurons through activation of NADPH oxidase. J Neurosci 24:565-575

24. Tong L, Balazs R, Thornton PL et al (2004) $\beta$-Amyloid peptide at sublethal concentrations downregulates brain-derived neurotrophic factor functions in cultured cortical neurons. J Neurosci 24:6799-6809.

25. Wang DC, Chen SS, Lee YC et al (2006) Amyloid- $\beta$ at sublethal level impairs BDNF-induced arc expression in cortical neurons. Neurosci Lett 398:78-82

26. Saez ET, Pehar M, Vargas MR et al (2006) Production of nerve growth factor by $\beta$-amyloid-stimulated astrocytes induces p75NTR-dependent tau hyperphosphorylation in cultured hippocampal neurons. J Neurosci Res 84:1098-1106

27. Roberson ED, Scearce-Levie K, Palop JJ et al (2007) Reducing endogenous tau ameliorates amyloid $\beta$-induced deficits in an Alzheimer's disease mouse model. Science 316:750-754

28. Baba A, Mitsumori K, Yamada MK et al (2003) $\beta$-Amyloid prevents excitotoxicity via recruitment of glial glutamate transporters. Naunyn Schmiedebergs Arch Pharmacol 368:234-238 